\title{
Development of Interactive Ophthalmology Hologram
}

\author{
Sarni Suhaila Rahim ${ }^{1}$, Nazreen Abdullasim², Wan \\ Sazli Nasaruddin Saifudin ${ }^{3}$ \\ Faculty of Information and Communication Technology, \\ Universiti Teknikal Malaysia Melaka \\ Hang Tuah Jaya, 76100 Durian Tunggal, Melaka, Malaysia
}

\author{
Raja Norliza Raja Omar ${ }^{4}$ \\ Department of Ophthalmology, Hospital Melaka, Jalan \\ Mufti Haji Khalil, 75400 Melaka, Malaysia
}

\begin{abstract}
Ophthalmology is one of the medical branches that deal with the eye. This field is associated with the anatomy, physiology and diseases of the eye. The main objective of this paper is to develop a novel interactive simulation of eye anatomy in three-dimensional (3D) by using holography environment approach in order to create better visualization of the structures of the eyes. Currently, public can access the information on medical through some conventional methods, such as brochure, pamphlet, booklet, in addition to some other better ways, for example 2D and 3D video. However, these methods do not offer an interactivity visualization of the medical information that help creating much engaging presentation to users. Moreover, the medical doctors are unable to show and explain in details about the diseases occurrence through these methods. An interactive method, therefore is required to assist the doctors to convey the disease information effectively. Since the human eye is the most complex organ in our body, thus an advanced technology, i.e., hologram should be used to visualize the visual system in an effective and interactive method, also to produce an effective eye disease explanation. Hologram is in a three-dimensional form besides an interactivity elements, where the proposed technology will help doctors examining vital organs using 3D displays. It is envisaged that the proposed interactive holography technique for clinical purpose would greatly contribute to and assist the management of the eye diseases and other diseases as well. It is hoped that the developed interactive holography technique for eye will assist clinicians in delivering the disease information efficiently and attractively.
\end{abstract}

Keywords-3D animation; hologram; ophthalmology; interactive; eye

\section{INTRODUCTION}

Holography was invented by Denis Gabor, a Hungarian physicist who found basic principles of microscopy while exploring to improve the transmission electron microscope efficiency in 1948 [1]. Recently, holography is a popular research area and many researchers focus on and contribute to the advancement of this study area. Most researchers focus on finding and proposing the technique or system through exploring the holography. Although there have been immense advancements in this area of research, there are still lacunae or spaces for improvement. The proposed techniques or systems in this research will most notably benefit the realm of holography in a number of areas or ways.
A study on the recent improvements in creating threedimensional images and videos by holographic techniques and the potential of holography in future is presented by Abbasi et al. [2]. Among the advantage of holography is the real 3D display without use of any other viewing aids and the quality of the science-art will be improved and as a result, it is impossible to differentiate between holographic images and the real objects [2]. In addition, in the field of art, Oliveira and Richardson [3] claim that the holographic technologies help change the cultural perception of artists and art institutions to hold holographic science. Moreover, the holographic technologies enable to create new dimensions and the likelihood to display a subject in 3D and beyond [3]. For example, one of the holographic features in Hologram Table [4], provides the display of digital models of cities or buildings as miniatures, with the ability to zoom in down to single blades of grass or even smaller.

This research aims to create an interactive hologram specifically for one of the medical branches, which is ophthalmology. There is a lack of interactivity visualization of the medical information through the conventional methods. In addition, through the conventional methods, such as brochure, pamphlet, booklet, or even 2D and 3D video, the presentation and explanation of diseases in details could not be conveyed effectively. Moreover, ophthalmology which deals with eyes, is consider an innovative and fast-moving field, where the human eye is the most complex organ in our body. Therefore, an advanced technology such as hologram should be used to visualize the visual system and produce eye disease explanation in an effective and interactive method.

The objectives of this paper are to study the holography method, to design and develop a novel 3D interactive simulation of eye anatomy by using holography environment approach in order to create better visualization of the structures of the eyes. Target users of this product are from different fields, such as for medical field, it can be used by the medical doctors, ophthalmologist and optometrists. In addition, for training field, the medical trainers or practitioners, nurses and paramedics are benefited from this research product. Besides, in education field, the medical lecturers, medical students, multimedia lecturers and multimedia students are benefited. Also, the public and patients can use this product as well. Currently, the research product focusing on one important module, which is Eye Anatomy. Methodology used for this 
research product is Multimedia Production Process which consists of three stages which are pre-production, production and post-production. The software used to create this product is Unreal. The outcome of this research is the interactive application of ophthalmology with holographic method. Besides, the research product provides the novel content verification for eye anatomy features by the Consultant Ophthalmologist, new verified human eye model and a novel voice-over recording for eye anatomy pronunciation by the Consultant Ophthalmologist. In addition, the research also received support, particularly in the contribution of expertise, from the Department of Ophthalmology at the Hospital Melaka, Malaysia.

This paper is organized as follows. Section 2 presents previous related work on hologram and interactive hologram system. Section 3 describes in details the overall methodology of this research. The discussion of the research paper is presented in Section 4. Finally, Section 5 presents some conclusions and future work.

\section{LITERATURE REVIEW}

The research product, Interactive Ophthalmology Hologram is an application developed using an engine called Interactive Hologram System. Interactive Hologram (i-HO) is an interactive system for viewing real-time 3D content on a holographic pyramid panel. This system consists of two parts, which are i-HO engine and i-HO projection panel. The novel development of an interactive hologram engine successfully overcome the limitation of traditional hologram, such as linear and video-based, no interaction and limited content representation. The developed system opens the possibility for creating an exciting 3D interactive content which can be used in various field, like games, education, architecture, engineering, medical and others. The interactive hologram architecture and process is presented in details in [5]. Fig. 1 shows the architecture of Interactive Hologram System, representing how input devices, $\mathrm{i}-\mathrm{HO}$ engine and the projection panel are being implemented.

The basic i-HO architecture consists of capturing images from real-time 3D content, mapping and projecting phases [5]. Hologram engine enable the user to interact in real-time with $3 \mathrm{D}$ content. The capturing part is a capturing process of a realtime 3D object from four different perspectives, which are frontal, back, left and right. Next, the captured images will be mapped into a single plane accordingly.

Interactivity is the main feature of $\mathrm{i}-\mathrm{HO}$ as it enables the developer to customize the engine for 3D game development and other $3 \mathrm{D}$ interactive contents. This important feature is different compared to many conventional hologram pyramid where the video or images were pre-rendered, linear and not interactive. Interactive hologram implemented provides interactive and immersive holography environment, in addition to real-time and interactive content in order to create better visualization. Therefore, with these capabilities offered by interactive hologram system, the developed Interactive Ophthalmology Hologram is able to produce better visualization of the eye model to show from different perspective includes frontal, back, left and right. In addition, it helps the model interacts with input devices, like keyboard, mouse, joystick or gamepad, sensor, motion capturing and motion controller. Moreover, the feature such as zoom in and out is provided to increase the usability efficiency of the product.

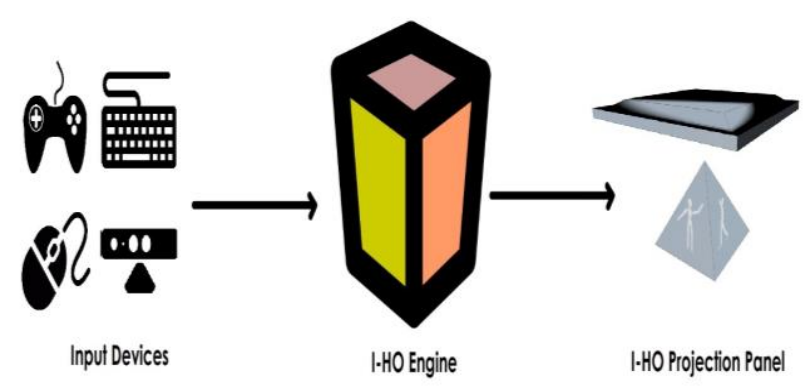

Fig. 1. Interactive Hologram System Architecture [5].

There are numerous medical application developed using holography. Holography in medicine and biology was presented earlier by Bally [6], comprising of holography in orthopaedic, radiology, ophthalmology, urology, dentistry, otology and others. Mehta [7] summarized the success of medical applications of holography shows a strong potential for holography to appear as a powerful tool for medical applications. Among the holographic techniques in medical are imaging through tissues, ophthalmology, dentistry, urology, otology, pathology and orthopaedics. For example, X-ray holography able to examine the samples in aqueous solution with high resolution without the sample preparation which end up with structural alternations [7]. Meanwhile, endoscopic holography provides a potential to record a 3D large focal depth and high resolution image of internal organs and tissues, hence enhances the capability of detection [7]. Besides, there are internal and external holographic endoscope which have been used for early detection of cancerous indurations in the wall of urinary bladder. Moreover, the multiplexed holography can be used for medical tomography which able to display the three-dimensional tomographic medical data. In addition, for imaging thorough human tissue, the holographic light-in-flight recording method can be adapted. In the ophthalmology field, the recording of a three dimensional eye image application was initially developed.

Recently in ophthalmology field, holography has potential to investigate corneal endothermal morphology, changes on the cornea, crystalline lens changes and the nerve head and the retina surface characteristics [7]. Potcoava summarized various holographic techniques and application on the imaging of ophthalmic tissue, fingerprints and microsphere samples [8]. Meanwhile, holographic in orthopaedics offers a benefit for the orthopaedic structures, especially external fixtures in order to reveal and measure strains on fixation pins and rods. Kim in [9] reviewed the digital holography focusing on the applications in biomedical microscopy, in addition to the related methods, current issues, trends and potential of holographic.

Based on holographic applications in medical presented above, it can be concluded that the developed application able 
to provide as a tool for visualizing patient data while training students and surgeons. It produces 3D real images and no special viewing devices or glasses are needed. Holographic applications in medical also allow the viewer to examine different organs or body parts, such as brain, liver, lungs, heart, skeleton, vascular system, nerves and muscles. The existing applications have covered different type of modules, different medical branches and different techniques. However, these existing systems do not offer an interactive content for development of the applications. Interactivity between the application and the user is the main issue or feature of holography. Therefore, the proposed research presents a novel interactive content for ophthalmology hologram, besides novel content verification for eye anatomy features, new verified Asian eye model and a novel voice-over eye anatomy pronunciation by the Consultant Ophthalmologist.

\section{METHODOLOGY}

The methodology used in this research is Multimedia Production Process, consists of three main phases, which are pre-production, production and post-production. There are six phases involved in these three main phases, which are analysis, design, implementation, testing, evaluation and publishing. In this section, all requirements will be further analysed in detail.

\section{A. Analysis}

In order to gather important information which is the content of the research, an interview session is conducted. The interview and discussion is conducted with the Consultant Ophthalmologist, Department of Ophthalmology, Hospital Melaka, Malaysia. The medical information is required in order to ensure the information provided is valid and sufficient for the user.

The data collection process can be divided into three phases. The first and second processes which are the content verification for eye features and verification on $3 \mathrm{D}$ eye model focusing on Asian eye model are conducted at the Eye Clinic, Department of Ophthalmology, Hospital Melaka, Malaysia. Meanwhile, the third process which is the eye anatomy voiceover recording in order to record the correct pronunciation of the eye anatomy for the research product is conducted at the Multimedia Studio, Faculty of Information and Communication Technology, Universiti Teknikal Malaysia Melaka. The recording is conducted in the studio due to the facility and the environment provided which suitable for the audio recording in order to produce the best output. Table I shows the details of data collection process. Meanwhile Table II, Table III and Table IV present the data collection summary for the first, second and third content verification process with the expert, respectively.

TABLE I. CONTENT VERIFICATION

\begin{tabular}{|l|l|}
\hline \multicolumn{1}{|c|}{ Verification } & \multicolumn{1}{|c|}{ Subject Matter Expert } \\
\hline Contents for eye anatomy features & \multirow{2}{*}{$\begin{array}{l}\text { Consultant Ophthalmologist, } \\
\text { Hospital Melaka }\end{array}$} \\
\cline { 1 - 2 } $\begin{array}{l}\text { Voice-over recording for eye anatomy } \\
\text { pronunciation }\end{array}$ & \\
\hline
\end{tabular}

TABLE II. CONTENT VERIFICATION SESSION 1

\begin{tabular}{|l|l|}
\hline Features & Description \\
\hline Location & $\begin{array}{l}\text { Eye Clinic, Department of Ophthalmology, Hospital } \\
\text { Melaka }\end{array}$ \\
\hline Date/Duration & 2 November 2017 \\
\hline Time & $3.00 \mathrm{pm}-5.00 \mathrm{pm}$ \\
\hline $\begin{array}{l}\text { Data Collection } \\
\text { Method }\end{array}$ & $\begin{array}{l}\text { Content Verification Session 1 } \\
\text { Discussion with the Consultant Ophthalmologist }\end{array}$ \\
\hline Discussion & $\begin{array}{l}\text { - Present 3D human eye model version 1 for expert } \\
\text { verification } \\
\text { - Explanation on the structures of eyes } \\
\text { - Once the eye model rotates, the labels of the eye } \\
\text { anatomy appear } \\
\text { - In addition to the glaucoma and diabetic retinopathy } \\
\text { age related macular degeneration can be added as } \\
\text { another eye disease }\end{array}$ \\
\hline
\end{tabular}

TABLE III. CONTENT VERIFICATION SESSION 2

\begin{tabular}{|c|c|}
\hline Features & Description \\
\hline Location & $\begin{array}{l}\text { Eye Clinic, Department of Ophthalmology, Hospital } \\
\text { Melaka }\end{array}$ \\
\hline Date/Duration & 11 November 2017 \\
\hline Time & $5.30 \mathrm{pm}-7.00 \mathrm{pm}$ \\
\hline $\begin{array}{l}\text { Data Collection } \\
\text { Method }\end{array}$ & $\begin{array}{l}\text { Content Verification Session } 2 \\
\text { Discussion with the Consultant Ophthalmologist }\end{array}$ \\
\hline Discussion & $\begin{array}{l}\text { - Present 3D human eye model version } 2 \text { for expert } \\
\text { verification } \\
\text { - Show the eye anatomy labelling } \\
\text { - Expert suggestions: } \\
\text { - Make the label line a bit tiny } \\
\text { - Add important anatomy, such as optic disc, ciliary } \\
\text { body, suspensory ligament } \\
\text { - Make a correction color for these anatomy: } \\
\text { Sclera (white) } \\
\text { Choroid (pink) } \\
\text { Retina (yellow) } \\
\text { - Follow the correct measurement for each of the } \\
\text { anatomy, must be proportional } \\
\text { - Make a correction on the design of lens (bigger) and } \\
\text { iris (a little bit curve) } \\
\text { - In addition to superior and inferior views, add lateral } \\
\text { view }\end{array}$ \\
\hline
\end{tabular}

TABLE IV. CONTENT VERIFICATION SESSION 3

\begin{tabular}{|l|l|}
\hline Features & Description \\
\hline Location & $\begin{array}{l}\text { Multimedia Studio, Faculty of Information and } \\
\text { Communication Technology, Universiti Teknikal } \\
\text { Malaysia Melaka }\end{array}$ \\
\hline Date/Duration & 3 April 2018 \\
\hline Time & $3.00 \mathrm{pm}-5.00 \mathrm{pm}$ \\
\hline $\begin{array}{l}\text { Data Collection } \\
\text { Method }\end{array}$ & $\begin{array}{l}\text { Content Verification Session 3 } \\
\text { Discussion with the Consultant Ophthalmologist }\end{array}$ \\
\hline Discussion & $\begin{array}{l}\text { - Audio recording for the Interactive Ophthalmology } \\
\text { Hologram introduction } \\
\text { - Eye anatomy pronunciation recording }\end{array}$ \\
\hline
\end{tabular}




\section{B. Design}

During the design phase, the product is designed to satisfy the requirements that identified in the previous analysis phase. In this phase, the human eye is modelled in 3D. The physical eye model is used as a guideline in order to model an accurate 3D eye model. In addition, the input from the Consultant Ophthalmologist also is considered throughout the design process of the 3D human eye model. The 3D human eye model is designed and developed according to the Asian human eye. This is another contribution of this research as other 3D human eye model is based on Caucasian eye. Fig. 2 shows the physical human eye model as a referral for designing the 3D human eye model. Meanwhile Fig. 3 shows the 3D human eye model referred. Fig. 4 to Fig 10 show some of the interfaces of this research product. Fig. 4 shows the interface of the eye anatomy module. Meanwhile, the interfaces of the eye anatomy which includes choroid and sclera are presented in Fig. 5 and Fig. 6 respectively. The interfaces of the eye cross section from four different views are shown in Fig. 7 to Fig. 10.

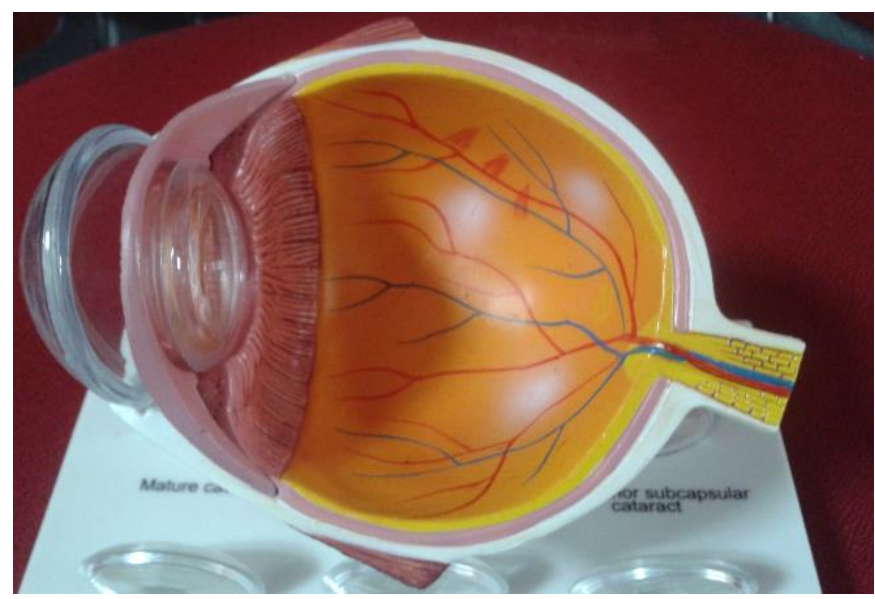

Fig. 2. Human Eye Model.

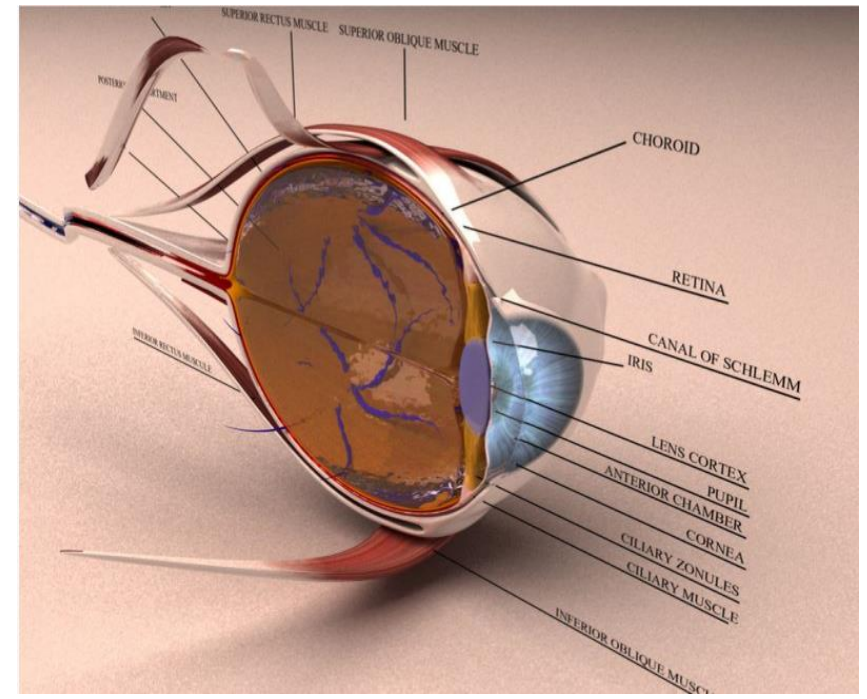

Fig. 3. 3D human Eye Model [10].

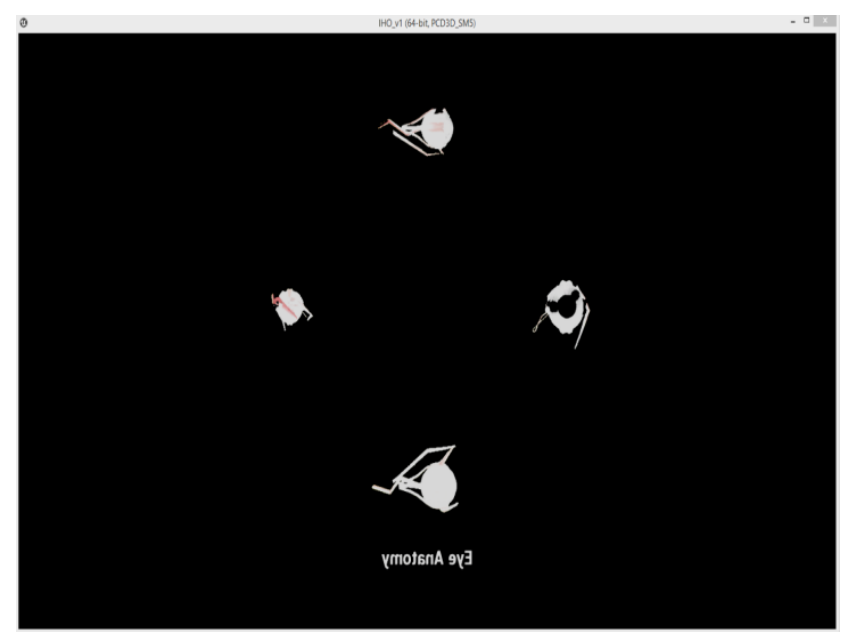

Fig. 4. Interface of Eye Anatomy Module.

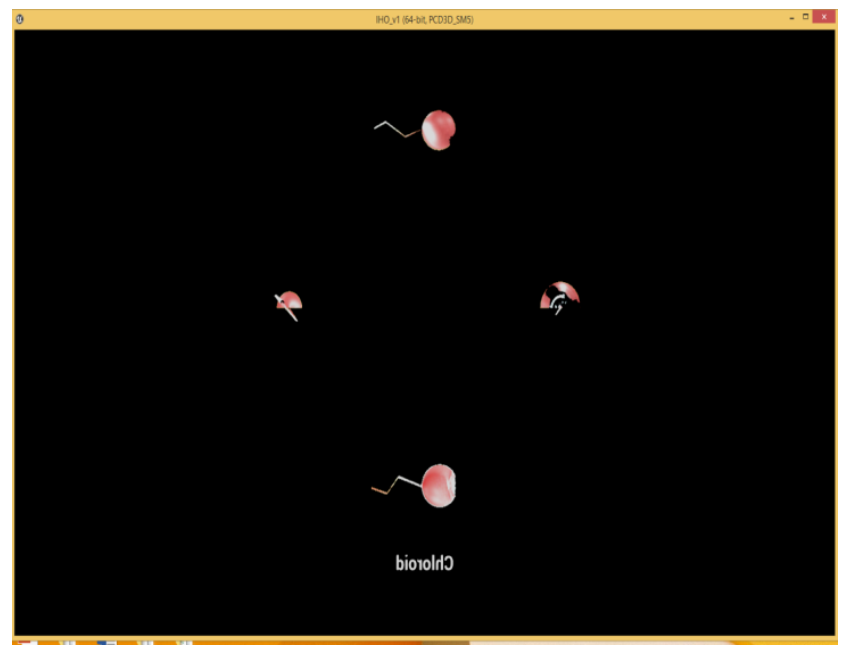

Fig. 5. Interface of Eye Anatomy Module - Choroid.

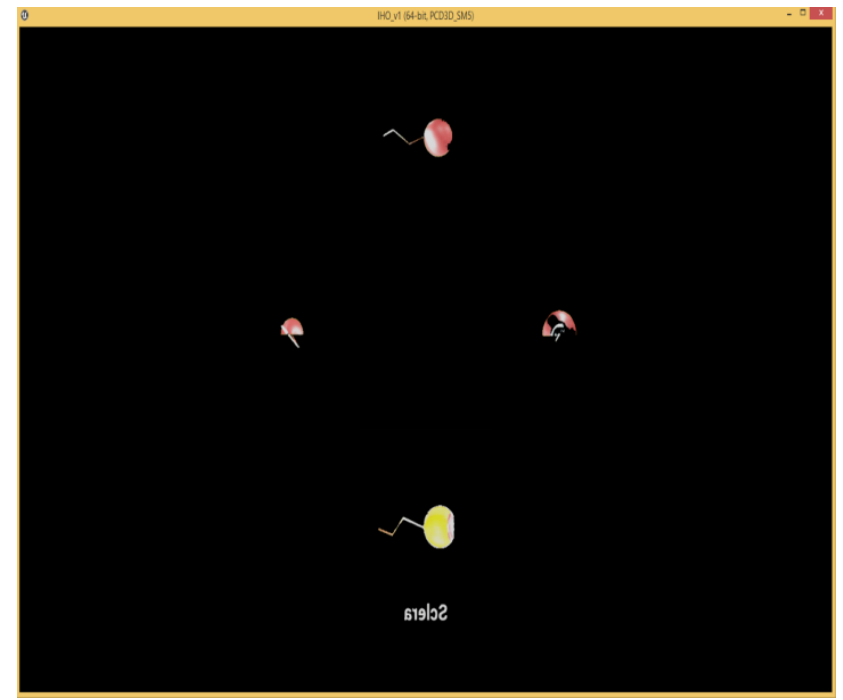

Fig. 6. Interface of Eye Anatomy Module - Sclera. 


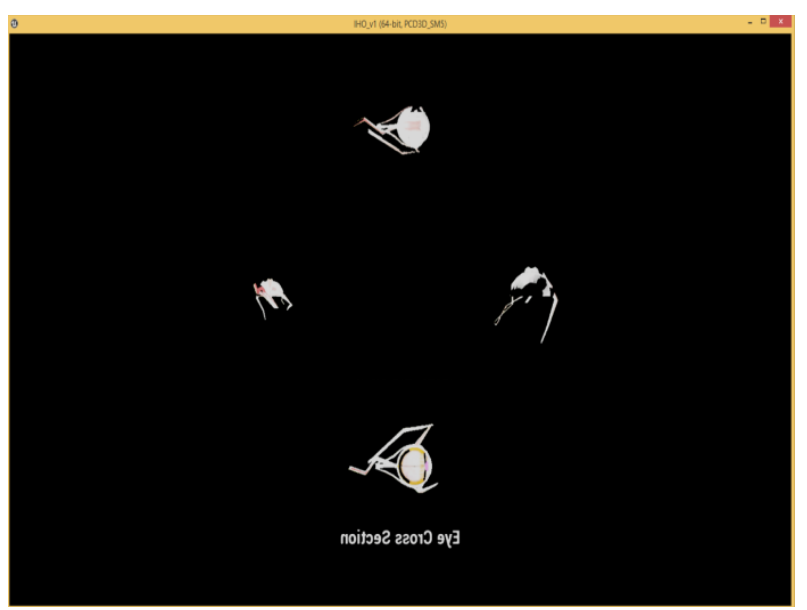

Fig. 7. Interface of Eye Cross Section View 1.

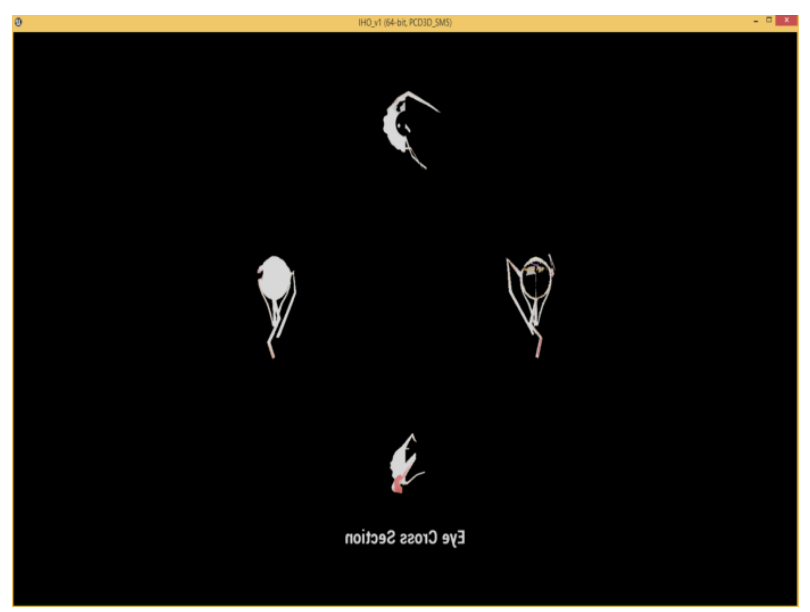

Fig. 8. Interface of Eye Cross Section view 2.

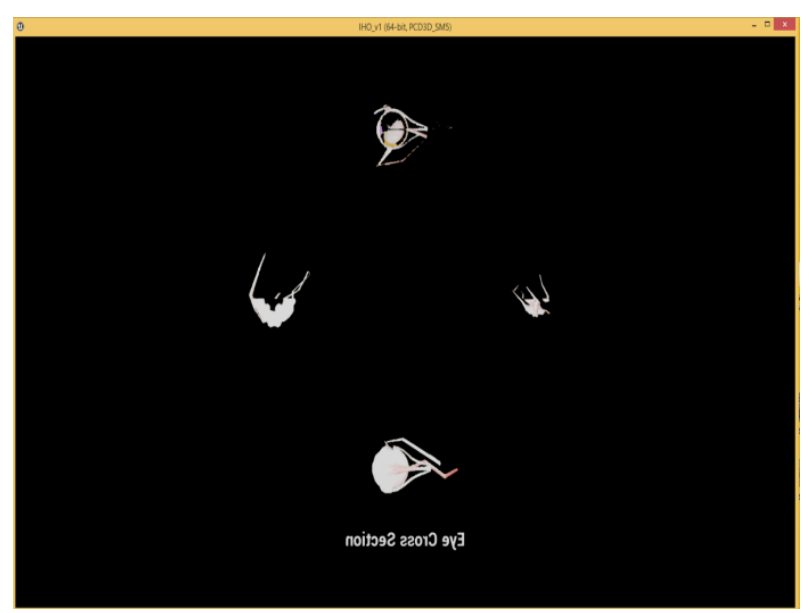

Fig. 9. Interface of Eye Cross Section View 3.

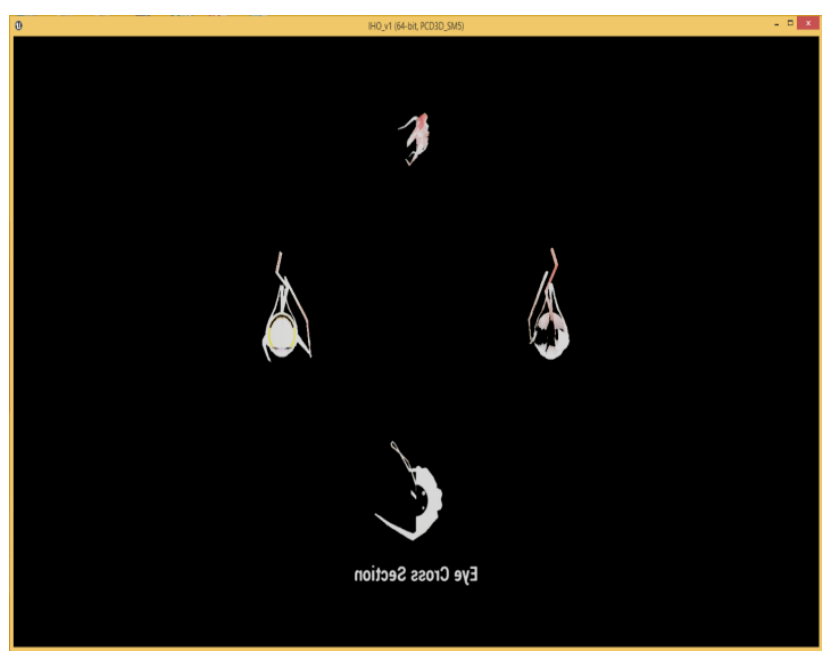

Fig. 10. Interface of Eye Cross Section View 4.

\section{Development}

Interactive Ophthalmology Hologram (O-iHO) has been developed by integrating a game engine with interactivity features. The product is capable to view the human eye anatomy from different perspectives, including the front, back, left and right sides. The human eye model also can be viewed in different cross section views. Besides representing the eye model in real time 3D graphic visualization, the product also able to provide real time interaction capability. The product can be interacted with devices such as keyboard, mouse, joystick and other input devices. Moreover, hand gesture and motion sensor are possible to interact with in order to produce an exciting experiential learning. The prototype of the product is presented in Fig. 11. Meanwhile, the product features developed are presented in Fig. 12 to Fig. 15. Fig. 12 shows the introduction screen of the Interactive Ophthalmology Hologram. Meanwhile, Fig. 13 presents one of the product features, which is the real time interaction capability. In addition, the other features which are interaction with hand gesture or motion sensor and also real time 3D graphic visualization are shown in Fig. 14 and Fig. 15 respectively.

The graphics or images used in this product are in 3D form to provide better visualization and details as the human eye is complex. The 3D image is designed by using Cinema 4D software. The audio is recorded by using audio recording equipment and then edited. The audio of the first page mentioning the title and the purpose of the product is provided once the user starts using the product. Meanwhile, the user can listen to the eye anatomy pronunciation for each of the eye structures every time when the eye features presented. The video of the proposed product which shows the implementation and details of the final output is presented in [11-12]. 


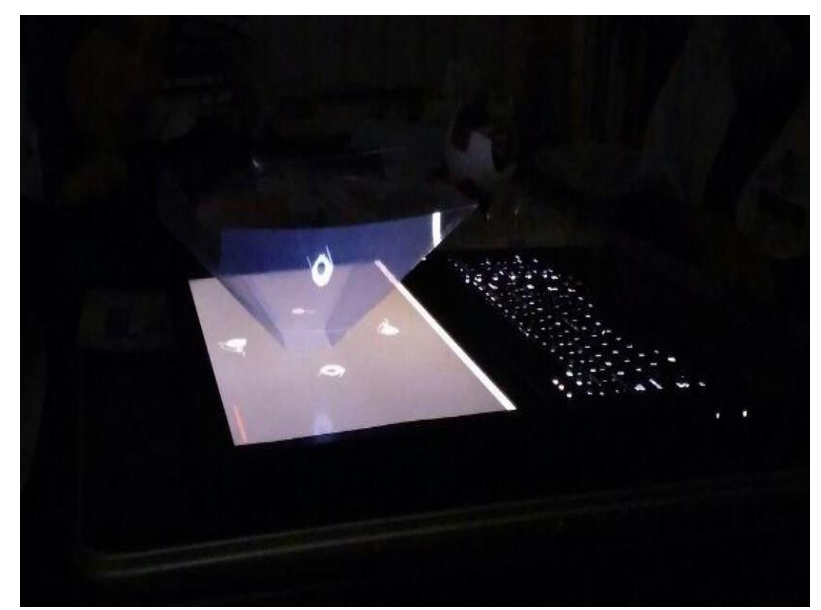

Fig. 11. Prototype Demonstration.

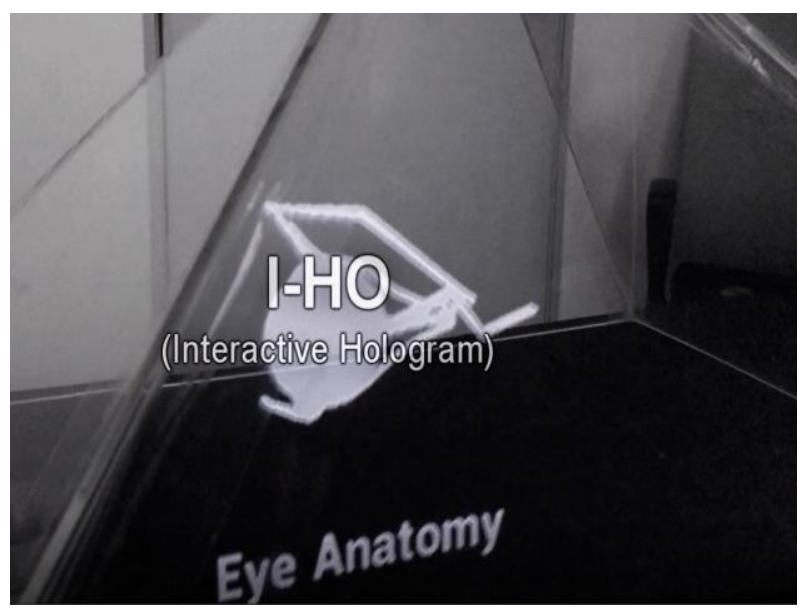

Fig. 12. Interactive Ophthalmology Hologram Introduction Screen.

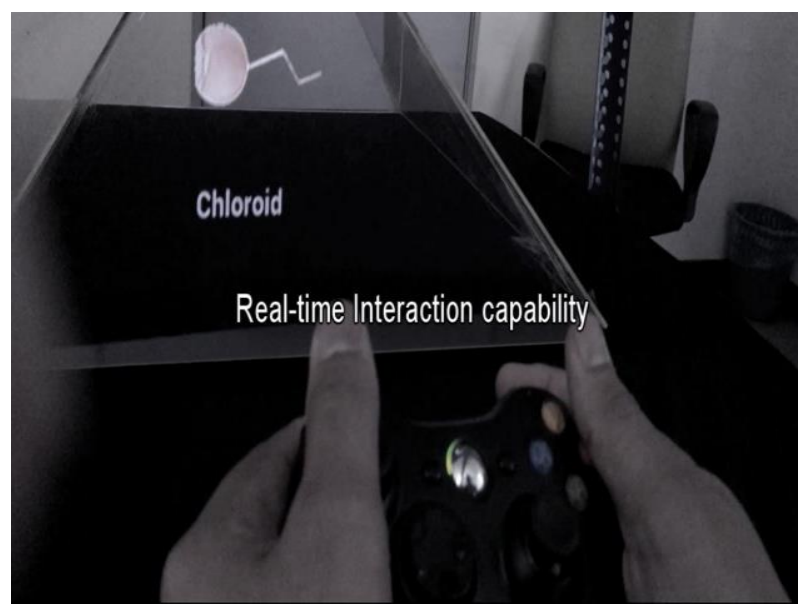

Fig. 13. Real Time Interaction Capability Feature.

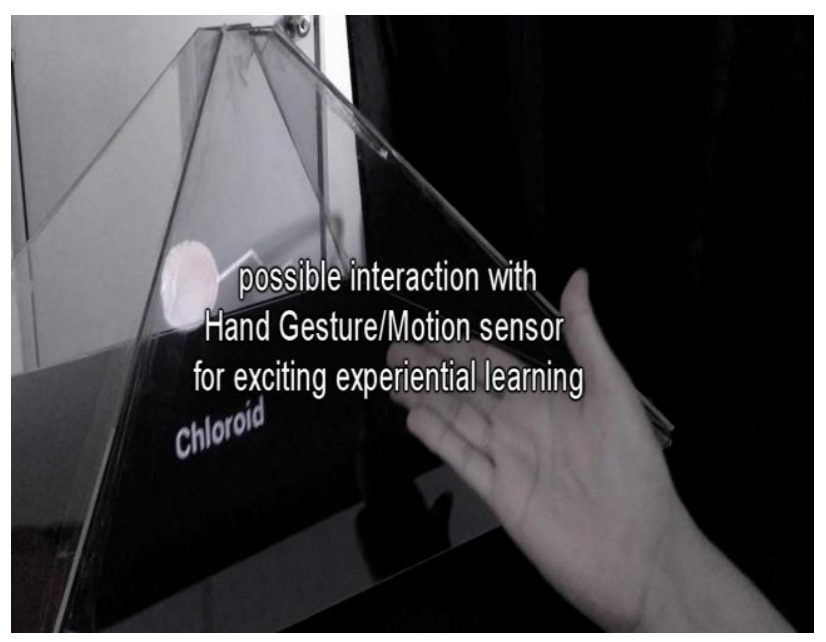

Fig. 14. Interaction Feature.

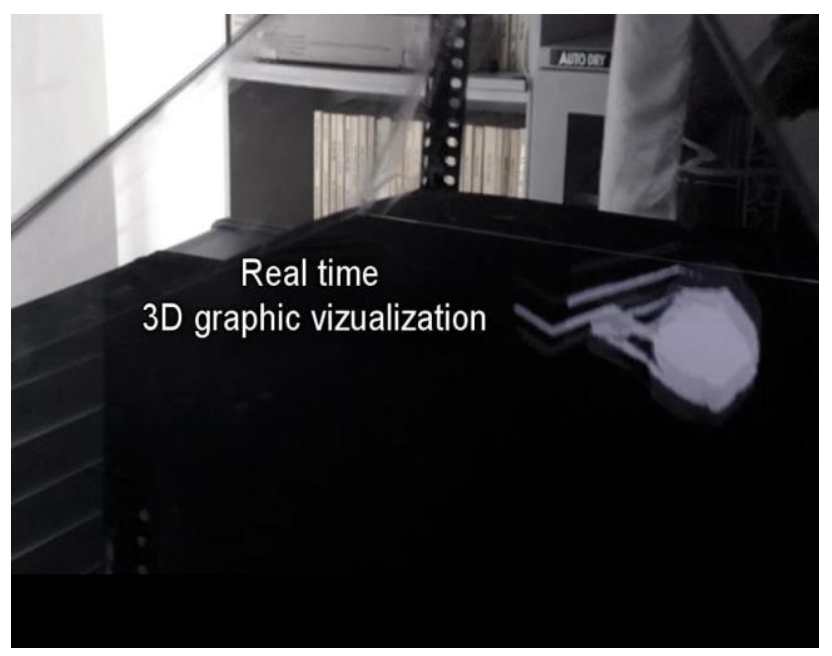

Fig. 15. Real Time 3D Graphic Visualization Feature.

\section{Evaluation}

Interactive Ophthalmology Hologram has been developed according to the objectives underlined. The product has been evaluated by the expert continuously. For every verification process, the product has been amended and improved based on the expert suggestions and comments in order to come up with a good output. The product has been presented to a big group of ophthalmologists during the National Glaucoma Update 2018 launching ceremony organized by the Department of Ophthalmology, Hospital Melaka, Malaysia on 27 April 2018 [12]. It was a great honoured to present the product to the target user and the responses received were positive and impressive. The product will be enhanced with other future improvements in order to provide a complete ophthalmology hologram. 


\section{DISCUSSION}

This research product is attractive as it consists of many multimedia elements and interactive capabilities, which able to attract the user attention. In addition, the characters of the animation are well designed for presenting the eye anatomy information. Moreover, the procedure of the product is designed nicely to make it ease of use for the users. The users can easily understand the information presented and how to interact with. The most important issue is the information presented is accurate since it is verified by the Subject Matter Expert which is the Consultant Ophthalmologist.

However, this research initially covers only one important module in ophthalmology field, which is the eye anatomy. By adding more modules, users can interact more with the application and their understanding towards ophthalmology such as eye anatomy and eye diseases can be increased. The explanation of each of the eye structures which have been verified by the expert will be added in order to provide more knowledge and understanding to the user. Moreover, users can easily understand each of the eye structures if the explanation is simple and understandable. The eye diseases module should be added for a complete content for ophthalmology hologram. Glaucoma, diabetic retinopathy and age related macular degeneration occurrence can be added as they are among the common eye disease. The holographic approach will help the medical doctors in conveying the information of the eye diseases, especially the complex signs of the eye diseases. For example, diabetic retinopathy is one of major complications of diabetes mellitus which causes blindness. Diabetic retinopathy is composed of a several of lesions found in the retina of individuals having diabetes. Therefore, the interest of this holographic approach could substitute current explanation procedures, eventually contribute to producing a more reliable and understandable explanation to the patients.

\section{CONCLUSION}

In conclusion, the interactive hologram for ophthalmology has developed successfully. The objectives have been achieved where the interactive content development provides the main ophthalmology information to the users. It is envisaged that the developed interactive hologram would greatly contribute to increase the understanding and awareness to the public especially patients. It is hoped that the developed application will assist the medical doctors, medical students and community in delivering the medical information efficiently.

This research contributes to the medical team, especially medical lecturers and medical students by increasing their understanding on eye anatomy and eye diseases. Furthermore, the product is not merely produced for the medical field, but also for education. Since holographic is a new advanced technology, the multimedia lecturers and students are benefited in exploring and learning this new technology. Moreover, this research provides a significant contribution to knowledge in the area of multimedia system. This is achieved by proposing a novel interactive hologram for ophthalmology.

\section{ACKNOWLEDGMENT}

This research is a collaboration between Universiti Teknikal Malaysia Melaka and Department of Ophthalmology, Hospital Melaka, Malaysia. This product won Gold Award Winner in the Innovation Carnival: UTeMEX 2017 organized by Universiti Teknikal Malaysia Melaka and Bronze Medal at the $29^{\text {th }}$ International Invention \& Innovation Exhibition (ITEX) 2018.

The product "Interactive Medical Hologram : Ophthalmology" is Universiti Teknikal Malaysia Melaka copyrighted (LY2018000965).

REFERENCES

[1] D. Gabor, "A New Microscopic Principle", Nature vol. 161, no. 4098, pp. $777-778,1948$

[2] H. Abbasi, T. Zalie, N. J. Farahani and A. G. Rad, "Studying the recent improvements in holograms for three-dimensional display", International Journal of Optics, vol. 2014, pp. 1-7, 2014

[3] S. Oliveira and M. Richardson, "The future of holographic technologies and their use by artists", J. Phys.: Conf. Ser., vol. 415, pp. 1-5, 2013.

[4] Euclideon Holographics, World's First Multi-User Hologram Table, available online: http://www.euclideonholographics.com, last visit 27.03.2018

[5] N. Abdullasim, W. S. N. Saifudin and S. S. Rahim, 'Development of interactive hologram system', Proceedings of Innovative Research and Industrial Dialogue 2018, 2018

[6] V. Bally, Holography on medicine and biology, Proceedings of the International Workshop, Munster, Fd. Rep. Of Germany, March 14-15, 1979, Springer-Verlag Berlin Heidelberg, 1979

[7] Integraf, Medical Applications of Holography, available online: https://www.integraf.com/resources/articles/a-medical-applications-ofholography, last visit: 27.10.2018

[8] M. C. Potcoava, Digital holography applications in ophthalmology, biometry, and optical trapping characterization, BiblioBazaar, South Carolina, United States, 198 pages, 2011

[9] M. K. Kim, "Applications of digital holography in medical microscopy", Journal of the Optical Society of Korea, vol. 14, no. 2, pp. 77-89, 2010

[10] TurboSquid, Human anatomy - eye section, available online: https://www.turbosquid.com/3d-models/3d-model-human-eye-section-/677870, last visit: $1.11 . .2017$

[11] Youtube, iHO: Interactive ophthalmology hologram, available online: https://www.youtube.com/watch?v=ysMPcXiaM8Q, last visit: 25.7.2018

[12] Youtube, iHO: Interactive ophthalmology hologram gimmick, available online: https://www.youtube.com/watch? $\mathrm{v}=\mathrm{ehV} 9 \mathrm{IWxgDS} 4$, last visit: 25.7.2018. 